\title{
PERFORMANCE OF AN ACTIVITY MONITOR INTEGRATED INTO A MICROPROCESSOR KNEE
}

\author{
Andy Sykes ${ }^{*}$, Nadine Stech ${ }^{1}$, Piotr Laszczak ${ }^{1}$, Michael McGrath ${ }^{1}$, Alan Kercher ${ }^{2}$, Saeed Zahedi ${ }^{1}$, David \\ Moser $^{1}$
}

${ }^{1}$ Endolite Technology Centre, Basingstoke, UK.

${ }^{2}$ Endolite North America, Miamisburg, OH, USA.

*Email: andy.sykes@blatchford.co.uk

\section{INTRODUCTION}

For many years, the biomechanical evaluation of prosthetic performance has centred around gait analysis and motion capture. While this provides useful, scientific insights, everyday life is not limited to straight-line, level, steady-state walking. With advancements in portable sensor technology in the last two decades, longterm activity monitoring (AM) has become a more feasible and reliable prospect for accurately representing the real-world walking behaviour of patients. Modern microprocessor knees (MPKs) have begun to have this functionality built into the devices themselves, without the necessity for additional, external hardware.

\section{METHODS}

This study aimed to validate the AM algorithm of one such MPK in a controlled laboratory environment. The participants were four transfemoral amputees (TFA) who regularly wore an MPK with in-built activity monitoring capabilities (Orion3, Endolite) day-to-day. A number of different activities were performed in order to test the robustness of the AM algorithm: six minute walk tests (6MWT) on a treadmill at self-selected slow, medium and fast speeds; walking in a figure of 8 ; walking clockwise and anti-clockwise around a $3 \mathrm{~m}$ square; walking up and down a ramp; walking up and down stairs. In order to provide validation for the algorithm, each participant wore a Stepwatch ${ }^{\mathrm{TM}} \mathrm{AM}$ around the pylon, above the prosthetic 'ankle'. All test sessions were video recorded and so a manual count from the video was taken as the 'gold standard' against which the two AM outputs would be compared.

\section{RESULTS}

An 'acceptable' performance for each device was determined to be within $10 \%$ of the manual count ${ }^{4}$. Both monitoring methods proved successful at achieving the target of $<10 \%$ error, with near perfect agreement with the manual, video-based step count. For the square and 'figure of 8' tests, the Stepwatch performed worse than it had during straight line walking, tending to overestimate the step count. The MPK algorithm performed very well during these tests, with $<5 \%$ error. During stair walking, the Stepwatch showed a good degree of accuracy, while the MPK was particularly susceptible to underestimation when gait compensation strategies were used (i.e. not walking step-over-step).
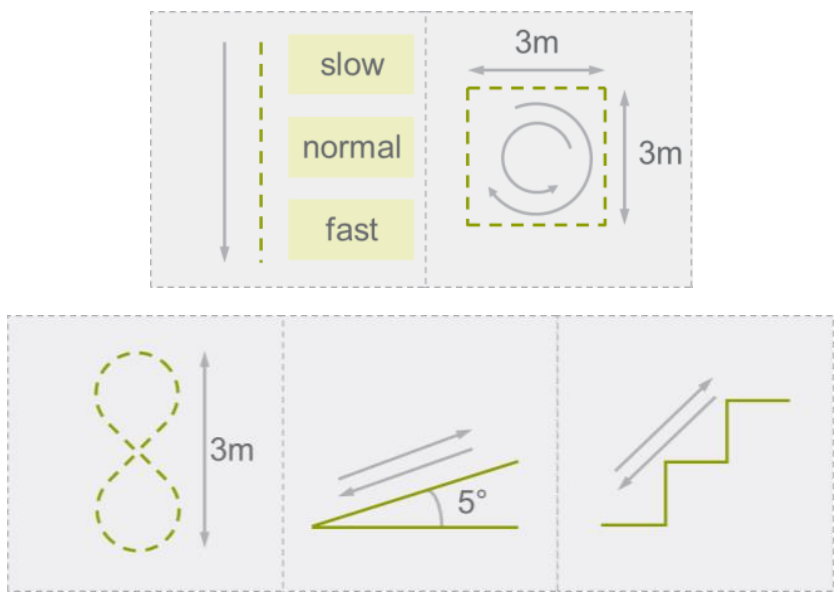

Figure 1: The activities performed for testing the step count algorithms

\section{CONCLUSION}

The performance of the in-built step counting algorithm has been shown to be comparable to that of the market leading, purpose-built hardware device, for a range of activities. While improvements are still possible, the data recorded by this algorithm could help prosthetists make informed prescriptions to best suit the needs of the patient, as well as supporting justification for the reimbursement of advanced prosthetic technology.

\section{SIGNIFICANCE}

This technology could allow remote monitoring of patient activity, potentially providing data to justify reimbursement of advanced prostheses. 


\section{REFERENCES}

1.Klute et al. Prosthetic intervention effects on activity of lower-extremity amputees. Arch Phys Med Rehab 2006; 87(5):717-22. DOI:10.1016/j.apmr.2006.02.007

2.Orendurff et al. How humans walk: bout duration, steps per bout, and rest duration. J. Rehabil. Res. Dev. 2008; 45(7):1077. DOI: 10.1682/JRRD.2007.11.0197

3.Arch et al. AAOP Annual Meeting and Scientific Symposium. Chicago, IL, USA. 1-4 March 2017.

4.Lee et al. Validity of consumer-based physical activity monitors. Medicine \& Science in Sports \& Exercise. 2014 Sep 1; 46(9):1840-8. DOI:10.1249/MSS.0000000000000287

\section{DISCLOSURE}

The Authors are employees of Endolite North America or Blatchford (the parent company of Endolite North America); the manufacturer of the MPK evaluated in this study. 